\title{
Scientific Program of NICA at JINR
}

\author{
V.D. Kekelidze*; A.D. Kovalenko, R. Lednicky, V.A. Matveev, I.N. Meshkov, \\ A.S. Sorin, G.T. Trubnikov \\ Joint Institute for Nuclear Research \\ E-mail: Vladimir.Kekelidzedcern.ch
}

\begin{abstract}
New scientific program is proposed at Joint Institute for Nuclear Research (JINR) in Dubna aimed to study hot and dense baryonic matter in the wide energy region from $2 \mathrm{GeV} / \mathrm{amu}$ to $\sqrt{s_{N N}}=11 \mathrm{GeV}$. To realize this program the development of JINR accelerator facility in high energy physics has started. This facility is based on the existing superconducting synchrotron Nuclotron. The program foresees both experiments at the beams extracted from the Nuclotron, and construction of heavy ion collider - the Nuclotron-based Ion Collider fAcility (NICA) which is designed to reach the required parameters with an average luminosity of $L=10^{27} \mathrm{~cm}^{-2} \mathrm{~s}^{-1}$. The spin physics program at NICA is developing as well.
\end{abstract}

36th International Conference on High Energy Physics,

July 4-11, 2012

Melbourne, Australia

\footnotetext{
*Speaker.

${ }^{\dagger}$ This work is supported by the RFBR, \#11-02-01538/12.
} 


\section{Introduction}

A study of hot and dense baryonic matter should shed light on: in-medium properties of hadrons and nuclear matter equation of state (EOS); onset of deconfinement (OD) and/or chiral symmetry restoration (CSR); phase transition (PT), mixed phase (see fig.1 left) and critical endpoint (CEP); possible local parity violation in strong interactions (LPV) $[1,2,3,4]$. It is indicated in series of theoretical works, in particular, in [3] that heavy ion collisions at $\sqrt{s_{N N}} \leq 11 \mathrm{GeV}$ allow to reach the highest possible baryon density in the lab (fig.1 right).

A project NICA aimed to study of hot and dense baryonic matter and spin physics is realizing at Joint Institute for Nuclear Research (JINR) in Dubna as a flagship project in HEP. In addition to the beams extracted from the Nuclotron (existing superconducting accelerator of variety of ions) the project foresees a construction of Nuclotron based Ion Collider fAcility (NICA) providing collision at the energy region up to $\sqrt{s_{N N}}=11 \mathrm{GeV}$ for $A u^{79+}$ with the luminosity $L=10^{27} \mathrm{~cm}^{-2} \mathrm{~s}^{-1}$.

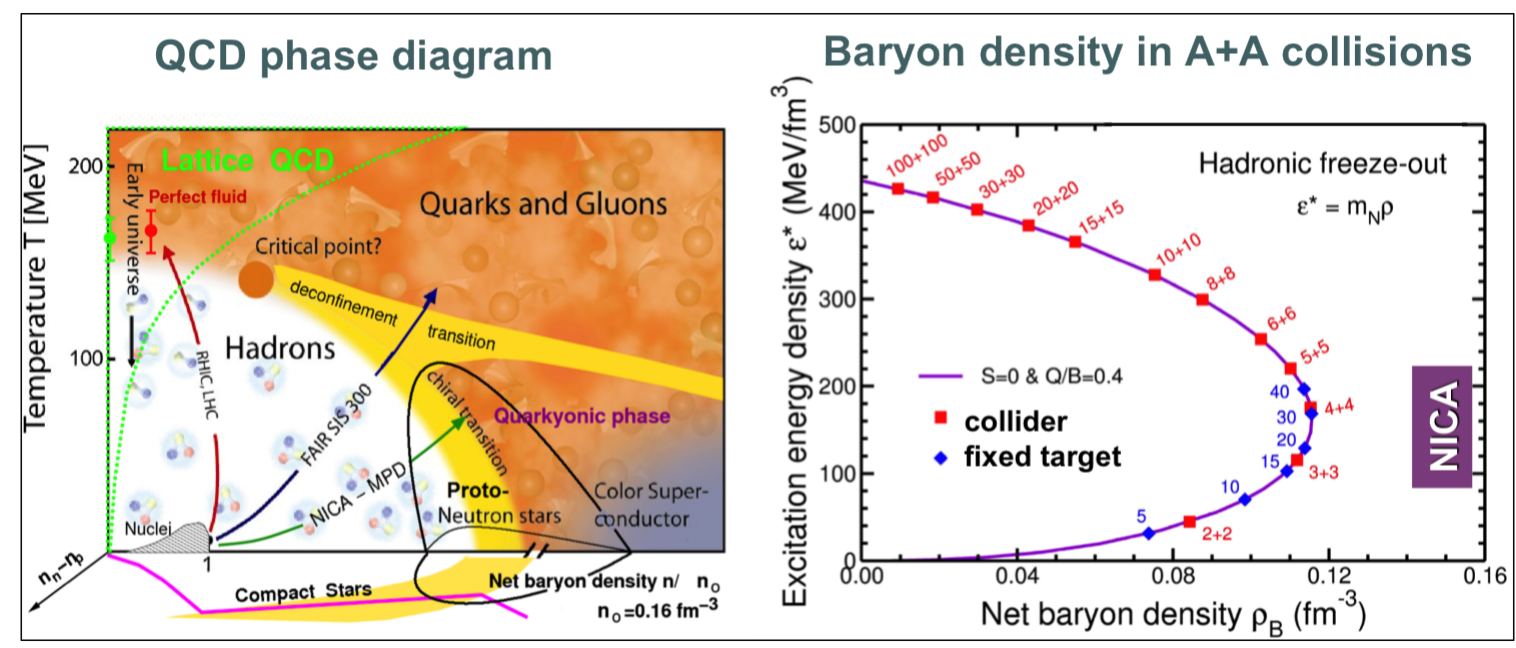

Figure 1: left: phase diagram for QCD matter (mixed phase is indicated by yellow); right: freezout diagrams for baryonic matter indicating baryon density reachable at different energies in collider and fixed target experiments [3] (the region covered by the NICA experiments is indicated).

The NICA will also provide the polarized proton and deuteron beams up to the c.m.s. energy of $27 \mathrm{GeV}$ for $p p$ collisions with the luminosity higher than $L=10^{30} \mathrm{~cm}^{-2} \mathrm{~s}^{-1}$. The high intensity and high polarization ( $>50 \%$ ) will provide a unique possibility for spin physics research, which is of crucial importance for the solution of the nucleon spin problem ("spin puzzle") - one of the main tasks of the modern hadron physics.

\section{Accelerator Facility NICA}

The Nuclotron - is an existing accelerator facility of JINR in HEP putting in operation in 1993. It is based on the unique technology of superconducting fast cycling magnets developed at JINR. The Nuclotron provides proton, polarized deuteron and multi charged ion beams.

The magnetic field of dipole magnets $B=1.8 T$ to the ion beam energies: $5.2 \mathrm{GeV} / u$ for d (A $=2, \mathrm{Z}=1) ; 3.3 \mathrm{GeV} / u$ for $\mathrm{Xe}(\mathrm{A}=124, \mathrm{Z}=42)$; and $4.05 \mathrm{GeV} / u$ for $\mathrm{Au}(\mathrm{A}=197, \mathrm{Z}=79)$. 
The new accelerator facility NICA includes: an injector complex providing wide spectrum of ions up to ${ }^{197} \mathrm{Au}^{32+}$ at energy $3.5 \mathrm{MeV} / u$ with an expected intensity $2 \cdot 10^{9}$; a booster accelerating ions up to $660 \mathrm{MeV} / \mathrm{u}$; the Nuclotron continuing acceleration up to maximum energy $(4.5 \mathrm{GeV} / \mathrm{u})$ and two storage rings with two interaction points (IP). The ions are fully stripped before the injection to the Nuclotron. The major parameters of NICA collider are following: $B \rho_{\max }=45 \mathrm{Tm}$; vacuum in a beam camera - $10^{-11}$ Torr; maximum dipole field $2 \mathrm{~T}$; kinetic energy from $1 \mathrm{GeV} / \mathrm{u}$ to $4.5 \mathrm{GeV} / u$ for $\mathrm{Au}^{79+}$; zero beam crossing angle at IP; $9 \mathrm{~m}$ space for each detector allocation at IP's; the reference luminosity for heavy ions $L=10^{27} \mathrm{~cm}^{-2} \mathrm{~s}^{-1}$. The required Nuclotron upgrade has started in 2008 and will be completed by 2015, including a booster and new linac. The overall construction schedule foresees that the storage ring and basic infrastructure facility should be available for the first ion collisions already in 2017 [5]. More detailed information is presented in [6].

The comparison of the NICA accelerator complex with the existing heavy ion machines and ones being in preparation is indicated in fig.2 (the energy scale is recalculated to the c.m. system related to the $\mathrm{Au}+\mathrm{Au}$ collision).

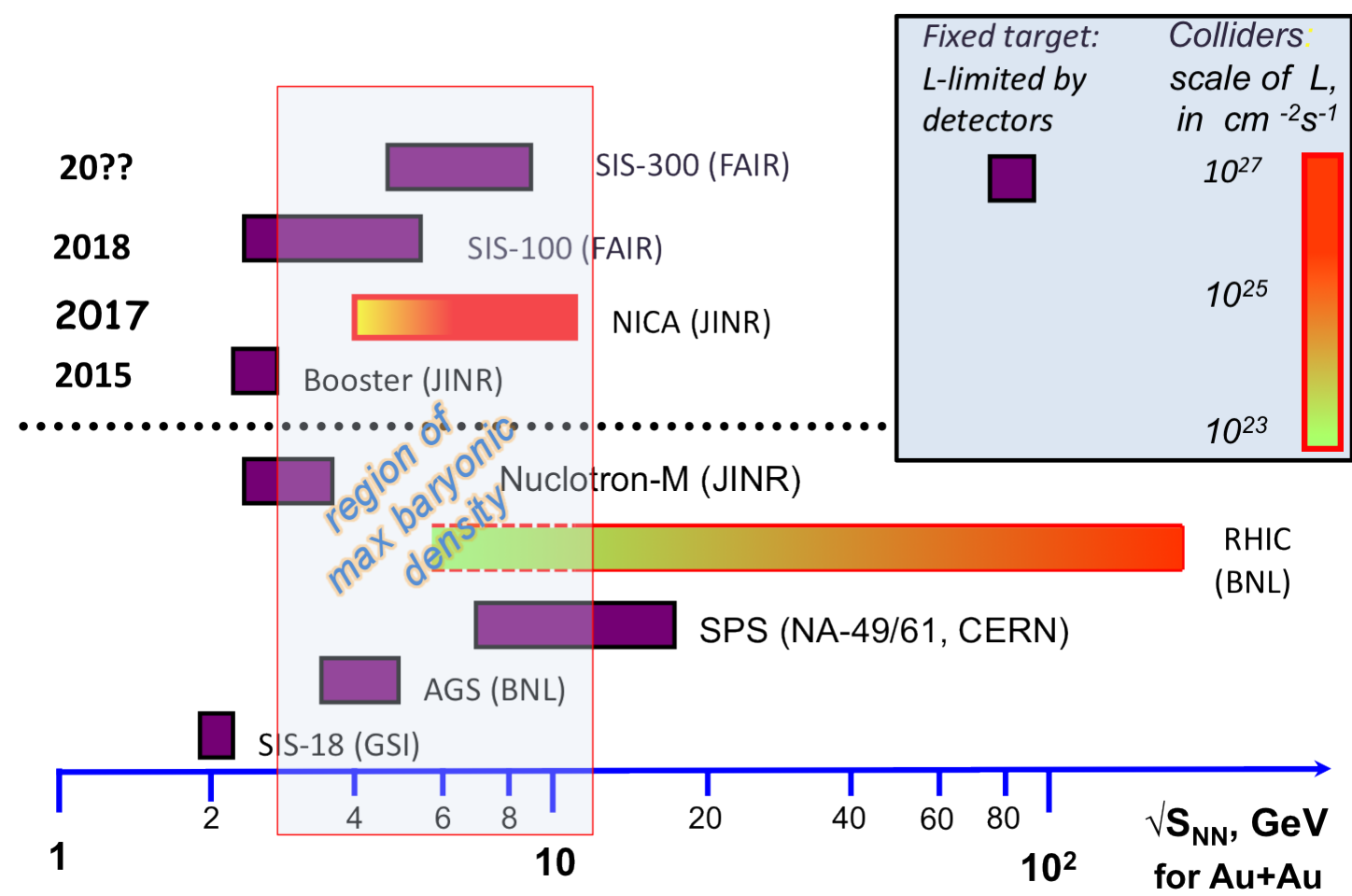

Figure 2: comparison of the running heavy on machines with ones being in construction (shadow in the frame indicates the region with maximum baryonic density)

In the first IP the MultiPurpose Detector (MPD) will be installed, while a detector for the second IP is not yet designed. A call for the corresponding proposal is announced.

\section{The BM@N Experiment}

The energy of the extracted beams provided by upgraded Nuclotron-NICA finally will be 
Table 1: The Nuclotron-NICA beams.

\begin{tabular}{|l|c|c|c|c|c|c|c|c|c|}
\hline beam & $\mathrm{p}$ & $\mathrm{d}$ & ${ }^{7} \mathrm{Li}$ & ${ }^{12} \mathrm{C}$ & ${ }^{24} \mathrm{Ar}$ & ${ }^{56} \mathrm{Fe}$ & ${ }^{84} \mathrm{Kr}$ & ${ }^{124} \mathrm{Xe}$ & ${ }^{197} \mathrm{Au}$ \\
\hline particles/pulse & $510^{12}$ & $510^{12}$ & $510^{11}$ & $210^{11}$ & $210^{11}$ & $510^{10}$ & $10^{9}$ & $10^{9}$ & $10^{9}$ \\
\hline
\end{tabular}

reached $6 \mathrm{GeV} / u$ for typical values of $\mathrm{A} / \mathrm{Z}=2$. A typical variety of possible beams and their intensities are presented in Table 1.

To realize the first stage of experiments at extracted beams with a fixed target a new setup - BM@N (Baryonic Matter at Nuclotron) will be constructed using existing wide aperture dipole magnet, tracking chambers, time of flight (TOF) system, hadron calorimeter and fast counter detector providing trigger signal. At the second stage an upgrade is foreseen to accomplish the setup with a silicon vertex detector (in cooperation with the partners from GSI, Darmstadt), with the electromagnetic calorimeter, and with the neutron detector (optional).

\section{The MPD Experiment}

The MPD experimental program is aimed at investigating both: the hot and dense baryonic matter, and the nuclon spin structure and polarization phenomena. A list of the first priority physics tasks to be performed in the experiment includes:

- in heavy ion program:

measurement of a large variety of signals at systematically changing conditions of collision (energy, centrality, system size) using as bulk observables the following:

- $4 \pi$ geometry particle yields (OD, EOS); - multi-strange hyperon yields and spectra (OD, EOS); - electromagnetic probes (CSR, OD); - azimuthal charged-particle correlations (LPV); - event-by-event fluctuation in hadron productions (CEP); - correlations involving $\pi, K, p, \Lambda$ (OD); - directed and elliptic flows for identified hadron species (EOS,OD);

reference data (i.e. $p+p$ ) will be taken at the same experimental conditions;

- in spin physics:

a study of polarization phenomena with polarized hyperons; a study of nuclon spin structure via the Drell-Yan (DY) processes.

The MPD experiment should be competitive and at the same time complementary to ones carried out at RHIC [7], and constructed in the framework of FAIR [8] project.

The MPD is a typical collider detector based on the solenoidal superconducting magnet. It will be installed in the first IP of NICA. The major sub-detectors of the MPD are (see fig. 3): solenoidal superconducting magnet with a magnetic field of $0.5 T$ ( $\sim 5 \mathrm{~m}$ in diameter and $\sim 8 \mathrm{~m}$ in length); time projection chamber (TPC); inner tracker (IT); time-of-flight (TOF) system; electromagnetic calorimeter (ECal); end cap tracker (ECT): and two forward spectrometers based on toroid magnets (optional). There are foreseen three stages of putting MPD into operation. The first stage of operation involves magnet, TPC, TOF, ECal (partially) and IT (partially), and should be ready for the first collision beams in 2017. 


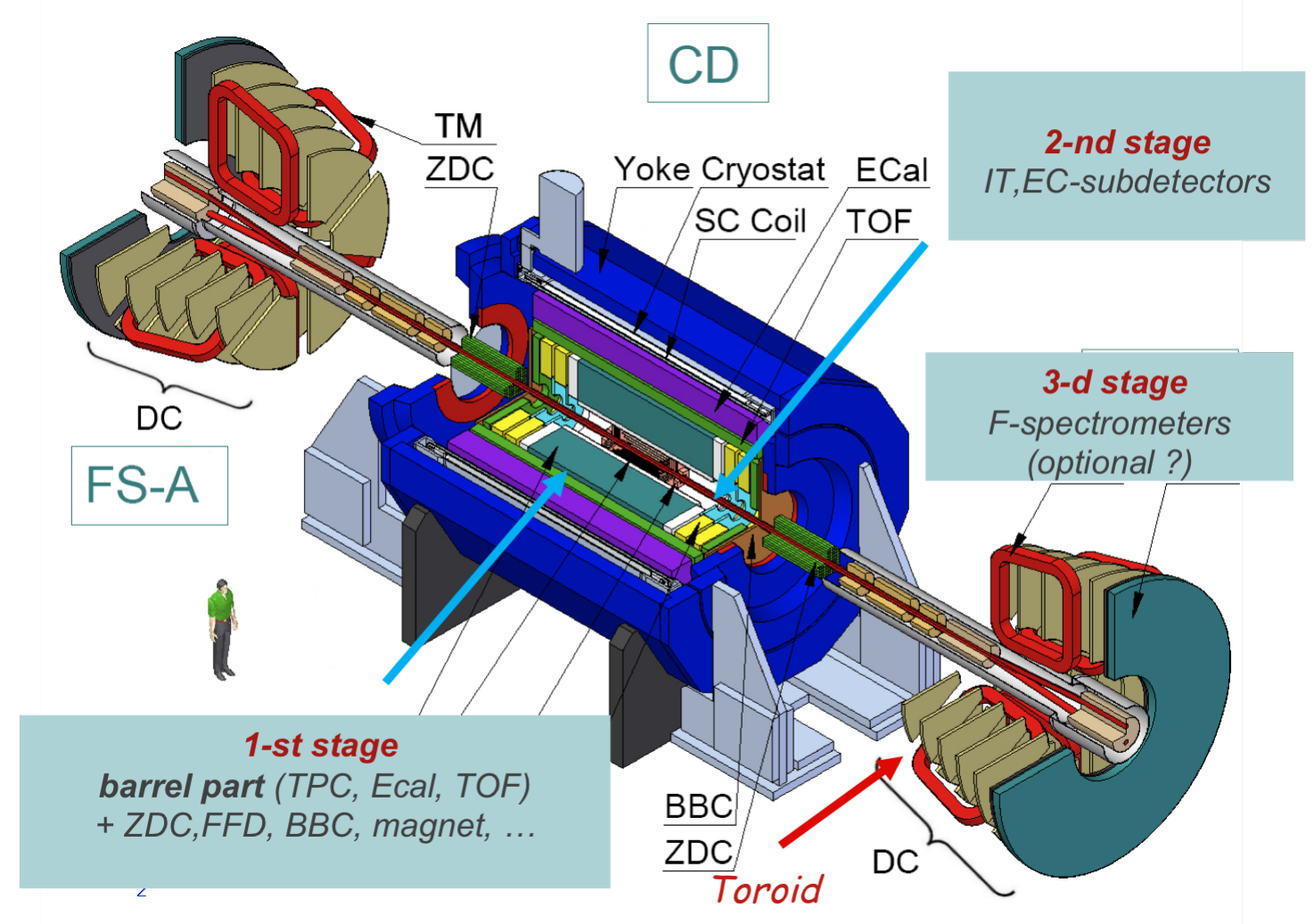

Figure 3: general view of the MPD, and sets of sub-detectors to be put in operation at different stages.

Processes studied with MPD were simulated using the dedicated software framework (MpdRoot). This software is based on the object- oriented framework FairRoot [9] and provides a powerful tool for detector performance studies, development of algorithms for reconstruction and physics analysis of the data. Evaluated rate in $\mathrm{Au}+\mathrm{Au}$ collisions at $\sqrt{s_{N N}}=7.1 \mathrm{GeV}(10 \%$ central interactions) taking into account the luminosity of $L=10^{27} \mathrm{~cm}^{-2} \mathrm{~s}^{-1}$ is $\sim 7 \mathrm{kHz}$.

More than ten working groups from 12 institutions are intensively working on sub-detector $\mathrm{R} \& \mathrm{D}$ and on prototyping of all detector elements. More detailed information could be found in the corresponding conceptual design report [10].

It was shown that MPD is well optimized ti study in-medium effects caused by high baryon densities, such as: changing particle properties in hot and dense medium (broadening of spectral functions etc.), event-by-event dynamical fluctuations of strange to non-strange particle ratios, and others. These studies could be done with better precision than those performed at present experiments. The simulations of MPD experiment shows that high statistics of studied events could be accumulated $\left(10^{9}\right.$ minimum bias events and $10^{8}$ central events per week) which provide the best precision for femtoscopy study with respect to RP and correlation of multistrange particles. In ten weeks of running more than $\sim 10^{7}$ of $\Omega$-hyperon decays will be recorded.

Charged particles are reliably identified using both techniques: measuring $d E / d x$ of tracks in TPC, and by TOF system (see fig.4). It was obtained sufficiently high resolution of vertices reconstruction illustrated in fig.5 (left). This figure (right) shows as well an example of $\Omega \rightarrow \Lambda K^{-}$ 
decay reconstruction implementing full chain of simulation: central $\mathrm{Au}+\mathrm{Au}$ collision generation at $\sqrt{s_{N N}}=7.1 \mathrm{GeV}$; hyperon productions and decays; decay product detection and their reconstruction using necessary MPD subdetectors.
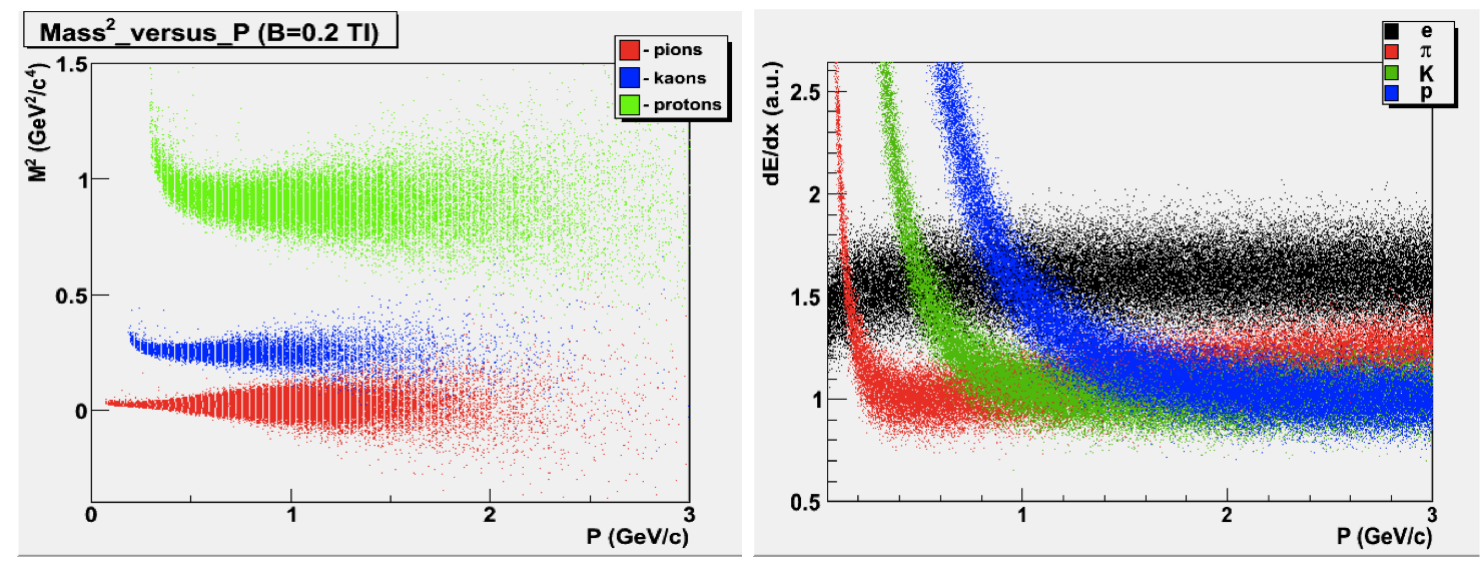

Figure 4: left: particle id using TOF system: right: particle id in the TPC by measuring the losses due to the ionization.

The MPD performance in general satisfies the required parameters for proposed experimental program. The further optimization of MPD element design is continued. The corresponding infrastructure is developed as well at the site in the Veksler and Baldin Laboratory of High Energy Physics (JINR, Dubna).
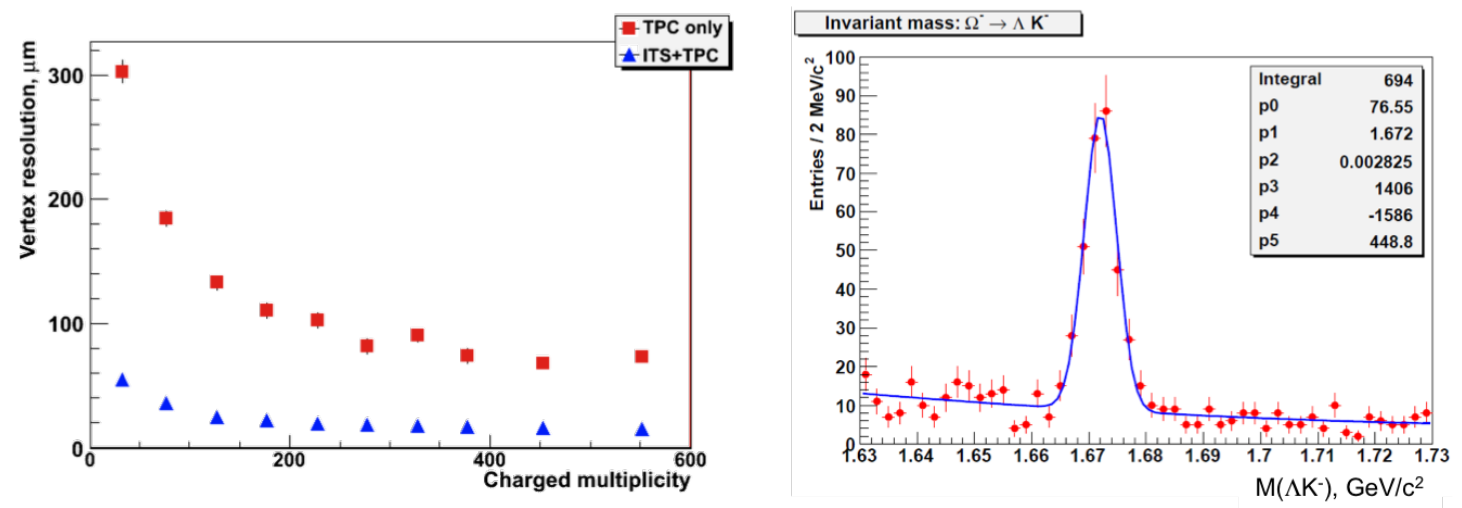

Figure 5: left: vertex resolutions versus multiplicity for events reconstructed with TPC only (squares), and for events reconstructed using both sub-detectors - TPC and IT (triangles); right: reconstructed invariant mass of $\Omega$ decay products (vertex reconstruction with TPC and IT, and particle ID with TPC and RPC)

\section{Detector for the second IP}

The NICA program foresees that a detector will be designed and installed in the second IP. The physics program of the related experiment should be dedicated, first of all, to the spin physics: a study of the Drell-Yan (DY) processes, not requiring the input from the poorly known fragmentation functions, which can be done in the kinematic region not available in other experiments. 
The creation of motivated collaboration has started. The proposal could be prepared and presented to the JINR scientific committees. The time scale of this experiment will be defined after the consideration of the corresponding proposal.

\section{References}

[1] A.N. Sissakian, A.S. Sorin, M.K. Suleimanov, V.D. Toneev, and G.M. Zinovjev, Part. Nucl. Lett. 5, 1 (2008).

[2] M. Gazdzicki, M. Gorenstein, and S. Mrowczynski, Phys. Lett. B585, 115 (2004).

[3] J. Cleymans, and J. Randrup, Phys.Rev.C74(2006)04791.

[4] B. Mohanty, J. Phys. G: Nucl. Part. Phys. 38 (2011) 124023.

[5] A. Sissakian et al., Design and Construction of Nuclotron-based Ion Collider fAcility (NICA) Conceptual Design Report (JINR, Dubna, 2008); http://www.nica.ru.

[6] V.G. Trubnikov et. al., XXXVI International Conference on High Energy Physics, Melbourne, 2012.

[7] G. S. F. Stephans, J. Phys. G32, S447 (2006), nucl-ex/0607030.

[8] P. Senger, J. Phys. G30, S1087 (2004).

[9] D. Bertini et al., J. Phys. Conf. Ser. 119, 032011 (2008), http://cbmroot.gsi.de.

[10] A. Sissakian et. al, Multi Purpose Detector Conceptual Design Report, v.1.2 (JINR, Dubna, 2010); http:// www.nica.ru. 